\title{
Application of optimal control theory on optimal advertising expenditure in monopoly
}

\author{
Nora Grisáková ${ }^{1, *}$, Peter Štetka ${ }^{2}$ \\ ${ }^{1}$ Department of Business Economics, Faculty of Business Management, University of Economics in \\ Bratislava, Dolnozemská st. 1/b, 82535 Bratislava, Slovakia (EU) \\ ${ }^{2}$ Department of Business Economics, Faculty of Business Management, University of Economics in \\ Bratislava, Dolnozemská st. 1/b, 82535 Bratislava, Slovakia (EU)
}

\begin{abstract}
Presented paper is being focused on Optimal control theory, Variation Calculus and its economic application. Aim of this research paper is to shortly describe Optimal control and Variation Calculus and to present how can we deal with these type of issues. The last part of this paper is presenting possible economic application of Optimal control, based on the maximization of profit in monopoly while introducing new product on the market. Our control variable is the advertising rate, which affects the profit of monopoly through advertising expenditures and as a state variable was the market share defined.
\end{abstract}

\section{INTRODUCTION}

Originally, the Optimal Control theory was a discipline focused on technical problems. But because of similarities between machine control and economic system management, optimal control theory was adopted by economists to solve economic problems. This adoption was very fast, because basic mathematic framework of optimal control theory was made without specific form of controlled system. Laščiak [1] says that optimal control is one that meets optimality criterion. Kamien and Schwartz [2] differ between management and decision making in optimal control. According to these authors, decision is a single action and management is an activity that directs system development over time. Optimal control is very close to variation calculus (we present the basics of this issue in next part of this paper). Both optimal problem theory and variation calculus are analytic methods of problems solving and planning in continuous time. Solution to the continuous time dynamic problem is a continuous function (or set of functions) indicating the optimal path to be followed by the variables through time (or space).

The indicators we want to put into the prescribed state, or we try to control, so these behave according selected criteria, are called system output and are denoted by $x(t)$. In the literature, so-called state variable is often mentioned instead of output. Control (or control variable) $u(t)$ is a variable that is being used to regulate the flow of control according to our criteria. Uncontrollable factors acting on the system are called failures and denoted as $p(t)$.

\footnotetext{
*Corresponding author: nora.grisakova@euba.sk
} 
We can define control system through mathematical means without specific interpretations. Lincoln [3] defines control system as following:

Let $P$ be a set of failure functions $p$ and let $U$ be a set of control functions $u$. System $S$ is an operator defined on the Cartesian space $P \times U$, assigning each pair $(p, u) \epsilon P \times U$ as function $x, x=S(p, u)$. In this case, the time, $t$, is represented in the operator $S$ only by $p$ and $u$, so it is an autonomous system. If the time in the operator $S$ acts as a separate variable, we are talking about a non-autonomous system.

If we use notation as a system, control variables or state variables we may imagine a machine. A lot of authors ([4], [5], [6], [7]) are using economic applications of optimal control theory. E.g. we may say that the national economy is a controlled system in the meaning of optimal control. State of national economy is indicated with a lot of economic indicators $x$. we try to set these indicators $x$ to desired values using economic management tools $u$. Similarly, a company can be controlled system that seeks to achieve its objectives $(x)$ with various tools $(u)$.

\section{OPTIMAL CONTROL PROBLEM AND VARIATION CALCULUS}

In optimal control problems, variables are divided into two kinds, state variables $x(t)$ and control variables $u(t)$ in defined time period $t_{0} \leq t \leq t_{1}$. State and control variables maximize objective function. The movement of state variable is governed by first order differential equations ${ }^{\dagger}$.

$$
\begin{gathered}
\max \int_{t_{0}}^{t_{1}} f(t, x(t), u(t)) d t \\
x^{\prime}(t)=g(t, x(t), u(t)) \\
x\left(t_{0}\right)=x_{0} \text { fixed, } x\left(t_{1}\right) \text { free }
\end{gathered}
$$

Where $f$ and $g$ are assumed to be continuously differentiable functions of three independent arguments, none of which is a derivative. The control variable $u(t)$ is a piecewise continuous function of time. The state variable $x(t)$ changes over time according to the differential equation (2) governing its movement. The control $u$ influences the objective function (1), both directly through its own value and indirectly through its impact on the evolution of the state variable $x$ (that also enter into the objective (1)). The highest derivative appearing in the problem formulation is the first derivative, and it appears only as the left side of the state equation (2). [2]

Optimal control problem could have several state and control variables. Each from state variables moves according differential equation (in continuous time period) or difference equation (in discrete time period). According to Dorfmann [8], differential equation condition presents the change rate in state variable in every time as a function of its present state and decision made $u(t)$. Number of control variables could be higher or lower than number of state variables.

If we define optimal control problem as in (1) - (3), then we need to find necessary conditions that lead to an optimal solution $u^{*}(t), x^{*}(t),\left(t_{0} \leq t \leq t_{1}\right)$. These necessary conditions finding is similar to solving nonlinear programming problem with Lagrange multiplier. Condition (2) of problem (1) - (3) must hold at every point in time $t$. We have function of Lagrangian multiplier $\lambda(t)$ from optimality conditions. Function $\lambda(t)$ is continuous by a differentiable function of time $t, t_{0} \leq t \leq t_{1}$.

\footnotetext{
$\dagger$ We denote derivative and partial derivatives by primes and subscripts. A prime label the derivative of the function with respect to its argument. Thus $f^{\prime}(x)=d f / d x, x^{\prime}(t)=$ $d x / d t, f^{\prime \prime}(x)=d^{2} f / d x^{2}, x^{\prime \prime}(g)=d^{2} x / d t^{2}$

* Star shows optimal solution of the problem in this paper.
} 
Sethi and Thompson say that the best way to find necessary conditions for function extreme is by applying Hamiltonian function. Work with this function is similar as with Lagrangian function for nonlinear programming problems (see example [9]). Before we start solving optimal control problem, we present fundamentals from variation calculus first. The reason is that optimal control theory comes from variation calculus and we need to apply it to some solutions [2].

\subsection{Fundamentals of Variation Calculus}

We can track the origin of variation calculus to Bernoulli [10] who was positing the brachistochrone problem and composed its solution. Later, other specific problems were solved and general theory was developed by Euler and Lagrange [11]. We can find the application of variation calculus in theoretical physics, in Hamilton's principle (for example described in [12], [13], [14], [15] and others) or the Principle of Least Action (described for example in [16], [17], [18] and others). The first economic applications were made in 1920s by Roos [19], Evans [20], Hotteling [21], Ramsey [22] and others.

This part of paper is intended only to present clarification of some parts of variation calculus which use the optimal control theory. For this reason we don't change the designation used in literature. Some designation in variation calculus could seem as in optimal control, but they could have different meaning.

Laščiak [1] says that variation calculus is a mathematical discipline which examines methods of finding functional extremes'. He formulated an easy variation calculus problem, where unknown function is time dependent.

Let $M$ be a set whose elements are functions. If we assign a single real number $I(x)$ to each functionx $\in M$, we say that the set $M$ is defined as a functional. The number $I(x)$ is called the value of the functional I on the function $x$ and the set $M$ is a set of permissible functions. [1]

Let be a function $F\left(t, x, x^{\prime}\right)$ that has continuous derivatives of at least the second order for $x \in\langle a, b\rangle$. From all curves $\mathrm{K}$ with the description $x=x(t)$, which have a continuous first derivative and pass through points $\left[a, x_{1}\right]$ and $\left[b, x_{2}\right]$, there must be found a curve for which the functional $\int_{a}^{b} F\left(t, x, x^{\prime}\right) d t$ has a minimum (maximum) value. We can formulate a necessary condition that this function must meet [2], [23]:

If the functional $\int_{a}^{b} F\left(t, x, x^{\prime}\right) d t$ takes extreme on the curve $K$ with the description $x=$ $x(t)$, then $x=x(t)$ satisfies the Euler equation $\frac{\partial F}{\partial x}-\frac{d}{d t} \frac{\partial F}{\partial x^{\prime}}=0$. According to Lagrange condition we find maximum or minimum of functional: If function $x=x(t)$ holds minimum (maximum) of functional $\int_{a}^{b} F\left(t, x, x^{\prime}\right) d t$, then $\frac{\partial^{2} F}{\partial x^{\prime} \partial x^{\prime}} \geq 0\left(\frac{\partial^{2} F}{\partial x^{\prime} \partial x^{\prime}} \leq 0\right)$.

Let's have a task of variation calculus

$$
\begin{aligned}
& \max _{x} \int_{t_{0}}^{t_{1}} F\left(t, x, x^{\prime}\right) d x \\
& x\left(t_{0}\right)=x_{0} ; x\left(t_{1}\right)=x_{1}
\end{aligned}
$$

For this task the Euler equation is

$$
F_{x}\left(t, x^{*}(t), x^{\prime *}(t)\right)=\frac{d F_{x}\left(t, x^{*}(t), x^{*}(t)\right)}{d t}, t_{0} \leq t \leq t_{1}
$$

or

$$
F_{x}=F_{x^{\prime} t}+F_{x^{\prime} x} x^{\prime}+F_{x^{\prime} x^{\prime}} x^{\prime \prime} t_{0} \leq t \leq t_{1}
$$

In equation (6) we've used a substitution $x^{\prime}=x^{\prime}(t), x^{\prime \prime}=x^{\prime \prime}(t)$. Euler equation is then second order differential equation and we can rewrite it through two first order differential equations

$$
p(t)=F_{x^{\prime}}\left(t, x, x^{\prime}\right)
$$

Now we can define new function - Hamiltonian function for the task (2):

$$
H(t, x, p)=-F\left(t, x, x^{\prime}\right)+p x
$$


Where $p$ is described from physics as general moment. In economic application $p$ could be interpreted as shadow price. We can write

$$
-\frac{\partial H}{\partial x}=p^{\prime} \quad \frac{\partial H}{\partial p}=x^{\prime}
$$

which represents canonical form of Euler equation [2].

\subsection{Necessary and sufficient conditions for the existence of extreme in optimal control}

In this part of paper we derive the necessary and sufficient condition for optimal control problem. The necessary condition for optimal solution $u^{*}(t), x^{*}(t), t_{0} \leq t \leq t_{1}$ of problem (4) comes from Hamiltonian

and its derivative

$$
H(t, x, u, \lambda)=f(t, x, u)+\lambda g(t, x, u)
$$

$$
\begin{gathered}
\frac{\partial H}{\partial u}=0 \rightarrow \frac{\partial H}{\partial u}=f_{u}+\lambda g_{u}=0 \\
-\frac{\partial H}{\partial x}=\lambda \rightarrow-\frac{\partial H}{\partial x}=-\left(f_{x}+\lambda g_{x}\right)=\lambda^{\prime} \\
\frac{\partial H}{\partial \lambda}=x^{\prime} \rightarrow \frac{\partial H}{\partial \lambda}=g=x^{\prime}
\end{gathered}
$$

From initial conditions we know that $x\left(t_{0}\right)=x_{0}$ and we assume $\lambda\left(t_{1}\right)=0$. In every $t$ is $u$ a stationary point of Hamiltonian for given value of $x$ and $\lambda$.Conditions (11) - (13) are necessary first order conditions for existing extrema for minimizing and maximizing problem.

Hamiltonian defined for optimal control problem (10) is similar to Hamiltonian defined for variation calculus (8). Also necessary conditions $-\partial H / \partial x=\lambda^{\prime}, \partial H / \partial \lambda=x^{\prime}$ are similar to canonic form of Euler equation (9). Second order necessary condition for maximizing problem says, that $H_{u u}\left(t, x^{*}, u^{*}, \lambda\right) \leq 0$ and for minimizing problem $H_{u u}\left(t, x^{*}, u^{*}, \lambda\right) \geq 0$.

If necessary conditions of optimality are also sufficient conditions, in nonlinear programing these conditions hold together when maximizing (minimizing) objective function is concave (convex) on closed convex set. We can have same results for variation calculus and dynamic optimization [2]. $\lambda$ could be interpreted as marginal value of continuous state variable $x(t)$ [24]. Extension of this process on more than one state variable $x$ and more control variable $u$ (number of state and control variable do not need be the same) could be find e.g. in [2] or [3].

\section{Optimal advertising expenditure for monopoly - Optimal control application}

Assume a company, which came to market with a new consumer durable product. This company is a monopoly till other companies do not come with similar product; therefore the company would like to maximize its profit from new product. We have to set a marketing plan during given time horizon in a way that total discounted profit from selling new product less advertising expenditures is maximized on diffusion constraint. We can formulate this model as:

$\S$ Multiplier $\lambda\left(t_{l}\right)$ represents so-called a transversal condition if there is no rescue value introduced in the model below. Detail description of these conditions shows for example Kamien and Schwartz [2]. 
Where

$$
\begin{gathered}
\max J=\int_{t_{0}}^{t_{1}} e^{-r t}\left[\left(p-c_{0}\left(\frac{x_{0}}{x}\right)^{z}\right) x^{\prime}-\left(a+b u+c u^{2}\right)\right] d t \\
x^{\prime}=g(x, u)=[\alpha+\beta u+(\gamma+\delta u) x](1-x) \\
x\left(t_{0}\right)=x_{0}
\end{gathered}
$$

$J$ total discounted monopoly profit from selling new product less advertising expenditures

$u$ advertising rate (control variable),

$x \quad$ reached market share (state variable),

$p \quad(p=100)$ constant price,

$\left(a+b u+c u^{2}\right)=A$ quadratic rate of market expenditures, $a, b, c$ nonnegative constants, $a=0, b=0.07, c=0.04$,

$r \quad(r=0.005)$ discount rate,

$z \quad(z=0.1)$ rate of decline in production costs,

$c_{0} \quad\left(c_{0}=70\right)$ constant production cost,

$x_{0} \quad\left(x_{0}=0.4\right)$ Initial market share of monopoly,

$\alpha \quad(\alpha=0.005)$ innovation coefficient,

$\beta \quad(\beta=0.006)$ coefficient related to the effectiveness of advertising towards innovators,

$\gamma \quad(\gamma=0.003)$ imitation coefficient,

$\delta \quad(\delta=0.007)$ coefficient related to the effectiveness of advertising towards imitators.

(time interval of new product $0 \leq t \leq 31-31$ week of new product advertising campaign)

We are using brackets to show each coefficient initial values for finding a solution. In this case we didn't use Hamiltonian in continuous time, but we've used algorithm presented by Teng and Thompson [25]. This algorithm changes continuous time in discrete one and for monopoly case we've made an assumption that there is only one company in the market. All the other assumptions and steps of algorithm remained same.

Presented model includes 13 parameters, one state (market share) and one control variable (advertising rate). From advertising rate we've calculate according function $\left(a+b u+c u^{2}\right)$ rate of advertising expenditures. As convergence criterion we've chosen to compare the absolute difference in objective function value in algorithm iterations. This absolute difference has to converge to zero.

In analyse we at first find the solutio $\mathrm{n}$ for initial values of coefficients and then we ceteris paribus change one of them.

\section{Discussion and Conclusion}

Optimal control theory and variation calculus were originally applied in purely technical disciplines. But these dynamics were very early used also in other disciplines, such as medicine, chemistry, economy etc. After summarizing basic notations and calculus from this field of mathematics, we've presented an economic application of optimal control theory. We've demonstrated it using a case of advertising expenditures optimization in monopoly. We've made 26 simulations, using selected initial values, with ceteris paribus rule being applied when changing our model parameters. Based on these simulations we've come to following findings:

- In all the cases with changed parameters, the same direction of a change in advertising rate $(u)$ and advertising expenditure rate $(A)$ was found.

- Increase in a "rate of decline in production cost" $(z)$ generates increase in all observed model variables.

- Increase in coefficient values in advertising rate function $(A)$ decreases company profit and monopoly market share. It also increases advertising rate $(u)$. 
- Change in discount rate $(r)$ causes opposite change in discount factor $\left(e^{-r}\right)$ and all other parameters of the model except one: the monopoly market share $(x)$, which changes in the same way as discount rate.

- Change in product price $(p)$ generates change of all the observed parameters in the same way.

- Change in a constant production cost $\left(c_{0}\right)$ generates opposite change in all other observed parameters.

- Initial market share $\left(x_{0}\right)$ change generates opposite change in all other parameters except one: total market share of monopoly at the end of observed period of time. This has the same direction as the initial market share.

- Innovation $(\alpha)$ and imitation $(\gamma)$ coefficient change generates the same direction change in monopoly market share $(x)$ and monopoly profit $(J)$. It also generates opposite changes in advertising rate and advertising expenditure rate $(A)$.

Changes in advertising effectiveness coefficient towards innovators $(\gamma)$ and imitators $(\delta)$ generate the same change in all model variables.

This paper is an outcome of research project VEGA 1/0646/20 „Diffusion and consequences of green innovations in imperfect competition markets ". Share of the project is $100 \%$. 


\section{References}

[1] Laščiak, A., Optimálne programovanie, Bratislava: Alfa, 1990, p. 597.

[2] Kamien, I. M., Schwartz, N. L., Dynamic Optimization: The Calculus of Variations and Optimal Control in Economics and Management, 2 ed., Dover: Dover Publication, 2012, p. 400.

[3] Lincoln, B., Dynamic Programming and Time-Varying Delay Systems, Lund: Lund Institute of Technology, 2003, p. 150.

[4] Goga, M., Ekonomická dynamika, Bratislava: Iura Edition, 2011.

[5] Yu, H., Bai, S., Chen, D., Dong, Ch., Feng, X., "Application of optimal control to the dynamic advertising decisions for supply chain with multiple delays," Systems Science \& Control Engineering, vol. 8, no. 1, pp. 141-152, 2020.

[6] Berberich, J., Köhler, J., Allgöwer, F., Müller, M., "Dissipativity properties in constrained optimal control: A computational approach," Automatica, no. 114, 2020.

[7] Zhang, J., Garcia Fracaro, S., Chmielewski, D.J., "Integrated Process Design and Control for Smart Grid Coordinated IGCC Power Plants Using Economic Linear Optimal Control," Processes, pp. 288-, 2020.

[8] Dorfmann, R., "An Economic Interpretation of Optimal Control Theory," The American Economic Review, no. 59, pp. 817-831, 1969.

[9] Fendek, M., Nelineárne optimalizačné modely a metódy, Bratislava: Ekonóm, 1998.

[10] Bernouli, J., "Problema novum ad cujus solutionem Mathematici invitantur," Acta Eruditorum, no. 18, p. from 269, June 1696.

[11] Thiele, R., "Euler and the Calculus of Variations," in Leonhard Euler: Life, Work and Legacy, Amsterdam, Elsevier, 2007, p. 249.

[12] Salmon, R., "Practical use of Hamilton's principle," Journal of Fluid Mechanics, no. 132, pp. 431-444, 1983.

[13] Pingyuan, W., Ying, Ch., Jinqiao, D., "Hamiltonian systems with Lévy noise: Symplecticity, Hamilton's principle and averaging principle," Physica D: Nonlinear Phenomena, no. 398, pp. 69-83, November 2019.

[14] Steinboeck, A., Saxinger, M., Kugi, A., "Hamilton's Principle for Material and Nonmaterial Control Volumes Using Lagrangian and Eulerian Description of Motion," Applied Mechanics Reviews, vol. 71, no. 1, pp. 1-14, January 2019.

[15] Jinkyu, J., Hyeonseok, L., Jinwon, S., "Extended framework of Hamilton's principle applied to Duffing oscillation," Applied Mathematics and Computation, no. 367, 15 February 2020.

[16] Feynman, R., Feynman's Thesis - A New Approach to Quantum Theory, London: World Science Publishing, 2005, p. 144.

[17] Siburg, K. F., The Principle of Least Action in Geometry and Dynamics, New York: Springer Verlag Berlin Heidelberg, 2004, p. 130.

[18] Litvinov, V., "Topological Magnetoelectric Effect," in Magnetism in Topological Insulators, Cham, Springer, 2020, pp. 79-89.

[19] Roos, C. F., "A Dynamic Theory of Economics," Journal of Political Economy, no. 35, pp. 632-656, 1927.

[20] Evans, G. C., "The Dynamics of Monopoly," American Mathematics Monthly, no. 31, pp. 77-83, February 1924. 
[21] Hotelling, H., "The Economics of Exhaustible Resources," Journal of Political Economy, pp. 137-175, April 1931.

[22] Ramsey, F., "Mathematical Theory of Savings," Economic Journal, vol. 38, no. 152, pp. 543-559, 1928.

[23] Tintner, G., "Monopoly Over Time," Econometrica, vol. 5, no. 2, pp. 160-170, 1937.

[24] Sengupta, J. K., Fox, K. A., Optimization Techniques in Quantitative Economic Models, Amsterdam: North - Holland Publishing, 1975.

[25] Teng, J. T., Thompson, G. L., "Oligopoly Models for Optimal Advertising when Production Cost Obey a Learning Curve," Management Science, no. 29, pp. 1087 1101, 1983. 